\section{Comparison of the Effects of Acupuncture and Acupunc- ture-Like Transcutaneous Electrical Nerve Stimulation Among Adults Nigerian with
Osteoarthritis of the Knee}

\author{
Sokunbi Ganiyu ${ }^{1 *}$ and Usman Mohammad Bello ${ }^{2}$ \\ ${ }^{1}$ Department of Physiotherapy, Faculty of Allied Health Sciences, Bayero \\ University, Kano State, Nigeria
}

${ }^{2}$ Department of Physiotherapy, Usman DanFodio University Teaching Hospital (UDUTH) Sokoto, Sokoto State, Nigeria

\begin{abstract}
Background: Osteoarthritis of the knee is a major cause of disability among adults with conservative therapies being among the preferred first line of treatment. Acupuncture and Transcutaneous Electrical Nerve Stimulation (TENS) are considered a potentially useful treatment for osteoarthritis, however there is dearth of information on the acceptability and efficacy of acupuncture in Nigeria.

Objectives: The study was designed to compare the effects of acupuncture and ACUTENS among adult Nigerian with OA of the knee joints.

Methods: Thirty patients with knee OA were randomised into 2 intervention groups (i.e., acupuncture and ACUTENS) and a control (soft tissue manipulation) group. Patients in the acupuncture and ACUTENS group were also treated with soft tissue manipulation as the control group. Pain intensity, functional mobility and knee Active Range of Movement (AROM) were measured at pre-intervention, post intervention and 3 months post intervention.

Results: Patients who received acupuncture showed statistically better improvement in the form higher mean changes \pm Standard Error of Means $(X \triangle \pm S E M)$ in pain intensity score of $4.20 \pm 0.01$ than the ACUTENS group who recorded changes in pain intensity scores of $30 \pm 0.30$. The mean changes in the functional mobility scores of $10.60 \pm 3.35$ and $4.30 \pm 1.12$ were recorded for the patients in the Acupuncture and ACUTENS group respectively. Also, the mean changes in the Knee active range of movement for the patients in
\end{abstract}

*Corresponding author: Sokunbi Ganiyu, Department of Physiotherapy, Faculty of Allied Health Sciences, Bayero University Kano, PMB 3011, Kano State, Nigeria, Tel: +234 08138468412; E-mail: ganiyusokunbi@gmail.com

Citation: Ganiyu S, Bello UM (2016) Comparison of the Effects of Acupuncture and Acupuncture-Like Transcutaneous Electrical Nerve Stimulation Among Adult Nigerian Adult with Osteoarthritis of the Knee. J Altern Complement Integr Med 2: 009

Received: November 30, 2015; Accepted: April 21, 2016; Published: May 06 2016 the acupuncture group was $15.5 \pm 5.18$ while patients who received ACUTENS recorded a mean changes of $5.50 \pm 1.38$. Acupuncture and ACUTENS treatment produced significant reduction in pain intensity level post intervention and at follow up assessments $(P<0.05)$. Patients who received acupuncture treatment showed significant improvement in functional mobility and knee active range of motion which was not present in the ACUTENS and control groups $(P<0.05)$.

Conclusion: Acupuncture and ACUTENS treatment had significantly better pain relieving effects when compared to soft tissue manipulation in patients with osteoarthritis of the knee. Acupuncture treatment produce better outcome than ACUTENS in terms of pain reduction, knee range of movements and functional mobility in patients with $O A$ of the knee.

Keywords: Acupuncture; Functional mobility; Knee Osteoarthritis; Pain; ACUTENS

\section{Introduction}

Osteoarthritis (OA) is the most common form of arthritis affecting $80 \%$ of those aged $65 y$ years or older [1,2]. The knee is the most vulnerable joint affected by OA [3]. There is a high prevalence of OA among adult populations [4]. It is estimated that approximately 15.8 million Americans have osteoarthritis of the knee [5]. In a Nigerian study, it was found that 229 out of 1403 participants, aged between 40 and 70 years reported knee OA, giving a prevalence of $16.3 \%$ [6]. $\mathrm{OA}$ of the knee is a common cause of severe, chronic, disabling and intractable pain [7]. The isometric peak torque of both the quadriceps and hamstrings of people with knee OA are weaker than those of individuals of the same age without knee OA [8]. In addition, gait velocity is slower, cadence is reduced, and stride length is shorter among people with knee OA [8]. After adjusting for age, sex, and comorbidity, knee osteoarthritis is responsible for a higher percentage of disability than any other medical condition for the following activities: stair climbing, walking a mile, and housekeeping [9]. Also, as the prevalence of osteoarthritis of the knee increases with age, it is possible that the already considerable impact of this disease will become even greater with the aging of the population [10].

No cure for osteoarthritis currently exists [11]. Thus, knee OA treatment focuses on managing the pain and dysfunction associated with the disease. Anti-inflammatory drugs usually, have various side-effects and patients with chronic knee OA pain increasingly seek alternative methods for pain relief [11]. Guidelines for the medical management of knee osteoarthritis by the American College of Rheumatology (ACR) in 1995 emphasize patient education, physiotherapy, occupational therapy, and exercise programs as the preferred first line non-pharmacologic methods of management of symptoms of knee OA [12]. The 2000 update of the ACR recommendations also mentioned acupuncture as a therapeutic approach under investigation [12]. Traditional Chinese Acupuncture (TCA) is based on the theory that vital energy, called 'qi', flows through the body along pathways called meridians [13]. There are specific points along these meridians, called acupuncture points, or acupoints, at which the de qi may be accessed. Inserting needles into these points permits restoration of harmony to the system, rebalancing the flow of de qi and to restore normalcy back to the body 
system [13]. In the Western medical model, acupuncture is thought to relieve knee OA pain through the gate-control mechanism or through the release of neurochemicals [14].

Acupuncture needle has been reported to activate sensory receptors in the muscles and this sends impulses to the spinal cord via type II and III muscle afferent nerves. Type II afferents were reported to signal the numbness of 'qi' needling sensation and type III, the fullness, heaviness and mild aching sensation [15]. Stux and Pomeranz [15] described the possible neural mechanisms of acupuncture analgesia as follows; small diameter muscle afferents are stimulated, sending impulses to the spinal cord, which then activates three centres (spinal cord, midbrain, and pituitary) to release neurochemicals (endorphins and monoamines) that block pain messages. Although the evidence supporting the endorphin hypothesis is overpowering, midbrain monoamines (serotonin and norepinephrine) are also involved in acupuncture analgesia; however, the role of the pituitary is less clear [15]. It appears that there is mixed evidence that acupuncture is an effective treatment of pain and physical dysfunction associated with osteoarthritis of the knee. Sangdee et al., [16] found that the reduction in pain was significantly greater for the acupuncture group versus the drug treatment (Diclofenac) group in a group of patients with OA of the knee. Tukamachi et al., [14] reported difference in pain levels between acupuncture group and drug treatment group but did not state whether or not the difference was significant. However, Sangdee et al., [16] reported that there was no statistically significant difference between acupuncture group and drug treatment group over time. The practice of acupuncture has not gained so much popularity, availability and acceptance in Nigeria unlike the use TENS.

Transcutaneous Electrical Nerve Stimulation (TENS) is one of the most widely used physical modalities for the management of OA of the knee. Similar to acupuncture, the effectiveness of TENS in the management of knee OA has been reported [17,18]. It has the advantage of being inexpensive, simple and essentially free of side effects. Transcutaneous electrical nerve stimulation may even be used at home by patients themselves due to its portability and simplicity. An essential feature of acupuncture like TENS (ACUTENS) is the use of strong stimulation to produce strong sensation to send impulses to the spinal cord and activates central nervous system to cause analgesia. Acupuncture like TENS parameters are stimulated at low frequency and high intensity $[17,18]$. In general, de qi occurs with current or voltage stimulation, which is 5-10 times the threshold value for muscle contraction. The intensity is kept below the level at which patient find it intolerable and hence that a pleasant mild aching sensation is felt. Although not completely understood the mechanism of action of acupuncture, it is safe and effective, recommended by the WHO (World Health Organization).

It appears that acupuncture is not yet popularized in Nigeria but in other countries, it is well recognized and valued for the control of pain and other treatments. Comparison of the efficacy of acupuncture and ACUTENS in the management of knee OA appeared not to have been widely investigated. Thus, the purpose of this study was to compare the effects of acupuncture and ACUTENS on pain, knee range of movement and functional mobility of patient with knee osteoarthritis.

\section{Materials and Methods}

\section{Design}

A Randomised Controlled Trial (RCT).

\section{Ethical approval}

Approval to carry out this study was obtained from the Research and Ethics Committee of the University of Maiduguri Teaching Hospital (UMTH), Maiduguri. Detailed information on what the study was all about, potential benefits and possible side effects and what will be expected of the participants during the study was provided in patient information sheet which was made available to the patient prior to the intervention. Participants were required to sign the written informed consent, and they were given enough time to consult with their doctors to decide whether they will be suitable to take part in this trial.

\section{Participants}

The participants for this study were males and females aged 45 years and above who have radiologically features, i.e., radiological alterations in the knee joint of grade 2 or more according to Kellgren-Lawrence criteria and clinically diagnosed with OA of the knee Joint according to American College of Rheumatology [19]. They are patients who has been certified by their doctors to meet the above conditions and were referred by their doctors to the physiotherapy clinics of UMTH, Maiduguri, Nigeria for physiotherapy treatment. Further inclusion criteria include knee pain lasting for 3 months or longer with no previous surgery to the knee joint and/or other co-morbid medical conditions such as benign or malignant tumours. Patients were also excluded if they had chondroprotective or intra-articular injection in the past 4 months, systemic corticoid treatment or, local antiphlogistic treatment in the past 4 weeks and acupuncture treatment during the past 12 months. Patients currently on analgesics at the time of the study were allowed to participate only if the medications had made no changes to their symptoms and there had been no changes in the dosage for at least 1 month prior to the commencement of this study. Patients with skin conditions that might impair skin sensation or prevent the use of TENS on the knee joints were also excluded.

Participant's flow chart is presented in figure 1.

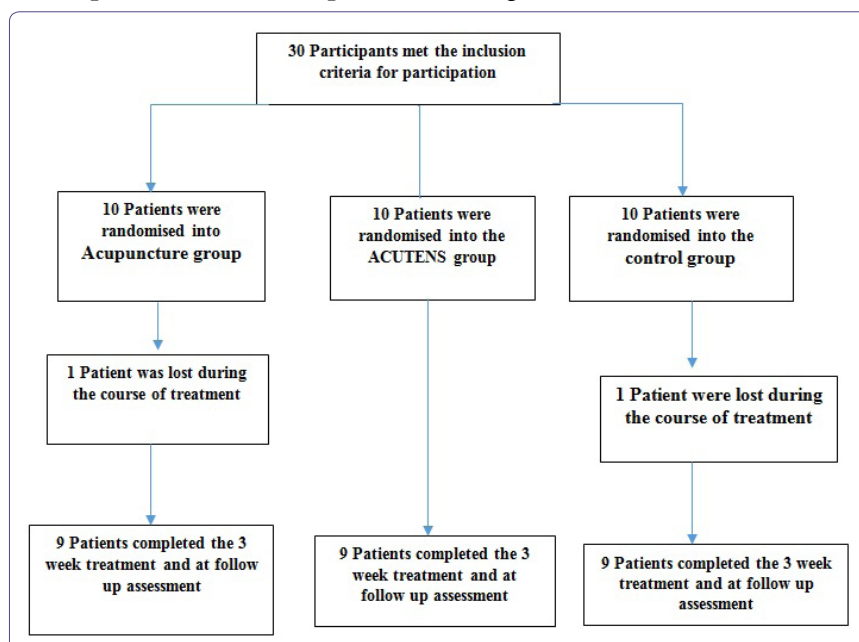

Figure 1: Participants flowchart.

\section{Sample size estimation}

Sample size estimation was based on the assumption that TENS could reduce pain intensity by at least 1.5 on Numeric Pain Rating Scale (NPRS) (this was based on the previous work which explored the effect of acupuncture and TENS in the treatment of knee OA) [20]. 
To yield a power of $80 \%$ with a significant level of 0.05 , a sample size of at least 8 subjects in each group was required (sample size estimation was determined by power analysis sample size software).

\section{Randomization}

A computer-generated randomization schedule was created to assign the participants into one of the 3 groups; acupuncture, ACUTENS and control (Soft Tissue Manipulation (STM)) groups. The groups were written on a pieces of paper and conceal inside a seal envelope until interventions were assigned. Ten participants were assigned into each group. Randomisation was carried out by a researcher while enrolment and assignment of the participants into the intervention group was carried out by another research assistant both of whom were not involve in any other aspects of the study. All participants were informed that they may be randomly allocated to any of the 2 intervention groups (Acupuncture + STM and ACUTENS+ STM) or the control group (STM). This procedure was carried out to ensure that each eligible participants have equal probability of being assigned to one of the 3 groups.

\section{Outcome measures}

Assessments of pain intensity, active range of knee flexion movement (active range of knee extension was not included as parts of the outcome measure as all the patients demonstrated full active range of knee extension at baseline assessment) and functional mobility were carried out prior to intervention, post intervention and at 4 weeks post-intervention follow up assessments by a researcher who was blinded to patients' group allocations.

Pain intensity was measured with Numerical Pain Rating Scale (NPRS). NPRS is a verbally administered scale that measures pain intensity $(0=$ "no pain at all" to $10=$ "worst possible pain"). Reliability and construct validity of NPRS with visual analogue scale was reported by von Baeyer et al., [21]. Active range of knee flexion was measured with universal goniometer with the participants in supine lying position. Universal goniometer was reported to have a validity of; $r=0.73$ and 0.77 when measuring angle of knee flexion [22]. Functional mobility was assessed with timed-up-and-go test. Participants were seated in chair with knee and hip joints maintained at $90^{\circ}$, feet in contact with the floor and with the forearm resting on the arm rest. The timed-up-and-go test was carried out as described by Nordin et al., [23]. The timed-up-and-go test has been validated and showed good intrarater and interrater reliability $(r=0.93$ and 0.96 , respectively) [23].

\section{Interventions}

Acupuncture intervention was provided by physiotherapist who was trained and certified by association of chattered society of physiotherapist, UK and with 6 years of experience in acupuncture treatment of musculoskeletal conditions. Patients were informed about acupuncture and ACUTENS in the study as follows: "In this study, different types of treatment will be compared. One type is similar to the acupuncture treatment used in China. The other types do not follow these principles, but has also been associated with positive outcomes in clinical studies". STM, acupuncture and ACUTENS treatments were carried out for 30 minutes, twice weekly and administered over 8 weeks period. For patients with bilateral knee osteoarthritis, STM, acupuncture and ACUTENS were administered to both knees. However, for patients with unilateral knee osteoarthritis, treatment was administered on the affected knee. Patients currently on analgesics (Non-Steroidal Anti-Inflammatory Drugs (NSAIDs)) at the time of the study were allowed to participate, only if the medications had made no changes to their symptoms and there had been no changes in the dosage for at least 1 month prior to the commencement of this study. If necessary, patients were allowed to continue to treat their NSAIDs they had been taking prior to the commencement of the study. The use of other pain treatments, such as drugs acting through the central nervous system, or corticosteroids, was not allowed as it could potentially have an effect on acupuncture and ACUTENS induced analgesia [18].

\section{Soft Tissue Manipulation (STM)}

Patients in all the groups (i.e., acupuncture, ACUTENS and control) received STM to the thigh and the knee joint area was administered to the participants in all the groups in this study. Patients are positioned in half sitting or supine lying position with proper pillow support at the back of the head and the knee for comfort. Techniques of soft tissue massage used were as described by Sokunbi and Usman [18]. STM was carried out for 5 min, twice weekly for 8 weeks.

\section{Acupuncture Treatment}

\section{Patient preparation}

Patients assume half lying supine position with proper pillow support under the head and neck and behind the knee joints for comfort. Also, they were informed of what to expect during and after the treatment in terms of the possible effects and side effects.

\section{Techniques}

Acupuncture treatment was carried out with the needles inserted at 3 selected points for the treatment of knee dysfunction according to the principles of Traditional Chinese Medicine (TCM), i.e., at the following acupoints; I) Neixiyan or EX-LE 4, located at the depression medial to the patellar ligament when the knee is flexed, II) Liangqiu on stomach meridian (Stomach 34), located at 2 finger breadth above the superior lateral border of the patella on the line connecting with the anterior superior iliac spine when the knee is fully flexed and III) Yinlingquan on spleen meridian (Spleen 9), located on the lower border of the medial condyle of the tibia on level with the tuberosity of the tibia (Figure 2) [15]. At each acupoint, the skin was wiped with alcohol, and the therapist's hands were clean with alcohol gel prior to the insertion of disposable stainless steel needles $(0.2 \mathrm{mmx} 40 \mathrm{~mm}$, Seirin, China). After insertion, the acupoints were manually stimulated by lifting, thrusting, twirling and rotating the needle, to elicit "de-qi". The needle manipulation was stopped when participants felt de "qi" ("de qi": numbness, soreness and or radiating sensation) [15]. The needle stimulation to elicit de qi was repeated at 5 minutes interval while the needles were left in position for 30 minutes. Treatments were carried out for 30 minutes duration twice weekly for 8 consecutive weeks.

\section{ACUTENS Treatment}

\section{Patient preparation}

Was as described for acupuncture group.

\section{Techniques}

Dual-channel TENS (Chinese, model 7000) was used to provide ACUTENS treatment. Electrodes were placed at similar locations as 


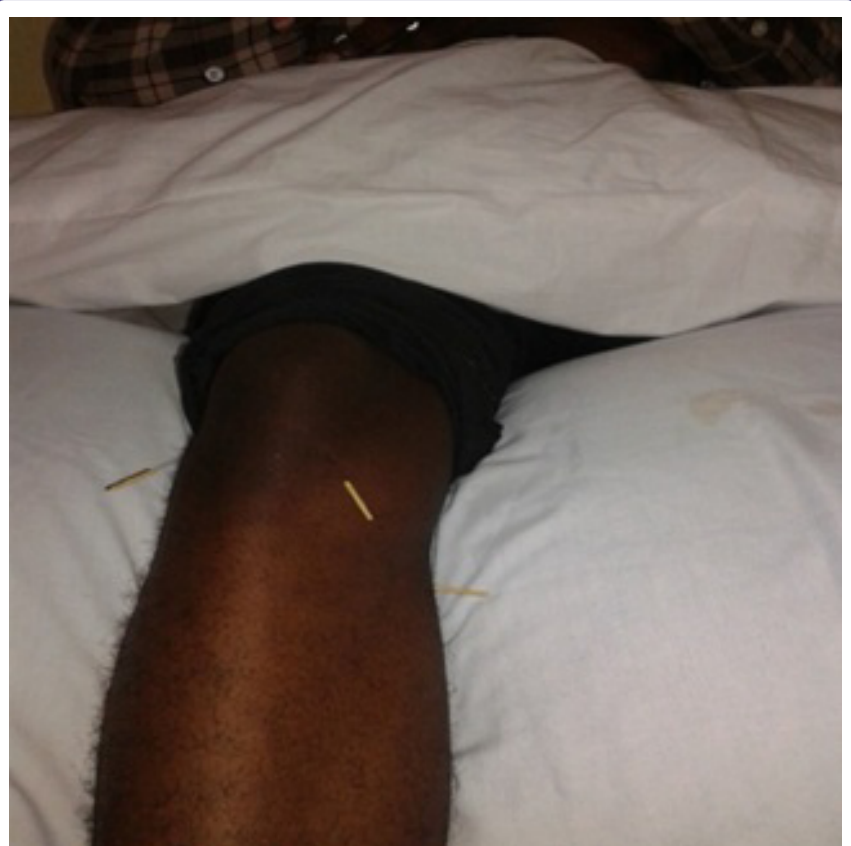

Figure 2: Acupuncture of ST 34, Spleen 9 and EX-LE 4.

for acupoints to as described above. The following TENS variables were used: Lowest rate/frequency possible $(2 \mathrm{~Hz})$, the highest intensity that were tolerated by the subject for $30 \mathrm{~min}$. Whenever the subject could tolerate the highest intensity stimulation, the duration of the pulse will be increased to the point of tolerance. Tolerance was defined as the level at which the patient asked the investigator to stop increasing the stimulation. Treatments were carried out for 30 minutes duration twice weekly for 8 consecutive weeks.

\section{Data analysis}

Statistical analyses were performed using Statistical Package for the Social Sciences (SPSS), version 14.0 based on intention-to-treat principle. Demographic variables such as age, weight, height and $\mathrm{BMI}$ and outcome variables such as pain, range of movements and functional mobility were presented as mean and Standard Deviation (X (SD)) while gender and patterns of knee OA were presented as frequency and percentage. Chi-square analysis was used to test for any significant difference in the gender and pattern of knee OA among the groups. One way Analysis of Variance (ANOVA) was used to compare age, weight, height and BMI among the three groups. Within group changes in the outcome variables over time period taking into consideration; pre intervention, post intervention and 3 months follow up were analysed with Multiple Analysis of Variance (MANOVA). Effects of intervention was analysed by comparing mean difference i.e., $\Delta \mathrm{X}$, (post-intervention mean score minuspre-intervention mean scores) of pain intensity, functional mobility and knee range of motion between Acupuncture and the ACUTENS groups. Statistical significance was set at 0.05 .

\section{Results}

Thirty participants were enrolled into the study and completed the 8 week intervention between March 2014 and January 2015. Three patients, one in each group were lost to follow up (One moved to another town, one car accident, and one reason unclear) (Figure 1). Thus, analysis of the data was carried out using intention-to-treat principle. Patients' age ranges from 49 years to 68 years. Thirteen
(43.3\%) of the participants were male while 17 (56.7\%) were female. Age BMI and duration of knee OA did not show significant difference among the groups $(\mathrm{P}>0.05)$ (Table 1). Table 2 shows significant reduction in the pre-intervention pain intensity level of the patients who received acupuncture and ACUTENS at post intervention and follow up assessments $(\mathrm{P}<0.05)$. Patients who received acupuncture treatment showed significant improvement in functional mobility and knee active range of motion which was not present in the ACUTENS and control groups $(\mathrm{P}<0.05)$. Analysis of the changes in the mean scores of pain intensity, functional mobility and active knee range of motion (mean post-intervention minus mean pre-intervention scores) between the acupuncture and ACUTENS group is presented in (Table 3). At 95\% CI, Patients who received acupuncture showed statistically better improvement in the form higher reduction in pain intensity, functional mobility and knee active range of movement than the ACUTENS group (Table 3).

\begin{tabular}{|c|c|c|c|c|c|}
\hline Acupuncture & \multicolumn{3}{|c|}{ ACUTENS } & \multicolumn{2}{c|}{ Control } \\
\hline Gender & $\mathbf{N ~ ( \% )}$ & $\mathbf{N}(\%)$ & $\mathbf{N}(\%)$ & $\mathbf{x}^{2}$ & $\mathbf{P}$ \\
\hline Male & $4(13.3)$ & $4(13.3)$ & $5(16.7)$ & 0.26 & 0.674 \\
\hline Female & $6(20)$ & $5(16.7)$ & $6(20)$ & & \\
\hline $\begin{array}{c}\text { Patterns of Knee } \\
\text { OA }\end{array}$ & & & & & \\
\hline $\begin{array}{c}\text { Unilateral Knee } \\
\text { OA }\end{array}$ & $5(16.7)$ & $6(20)$ & $5(16.7)$ & 4.10 & 0.237 \\
\hline Bilateral knee OA & $5(16.7)$ & $5(16.7)$ & $3(10)$ & & \\
\hline $\begin{array}{c}\text { Age (years) } \\
\text { BMD) }\end{array}$ & $58.11(10.3)$ & $\mathbf{X}(\mathbf{S D})$ & $\mathbf{X}(\mathbf{S D})$ & $\mathbf{F}$ & $\mathbf{P}$ \\
\hline BMI (kgm-2) & $29.4(4.79)$ & $30.23(5.50)$ & $58.67(10.9)$ & 5.124 & 0.16 \\
\hline $\begin{array}{c}\text { Duration of knee } \\
\text { OA (years) }\end{array}$ & $6.52(0.81)$ & $7.45(0.86)$ & $5.57(1.45)$ & 0.981 & 0.135 \\
\hline
\end{tabular}

Table 1: Demographic characteristics of the participants.

\begin{tabular}{|c|c|c|c|c|c|}
\hline & $\begin{array}{c}\text { Pre } \\
\text { treatment } \\
\text { X (SD) }\end{array}$ & $\begin{array}{l}\text { Post } \\
\text { treatment } X \\
\text { (SD) }\end{array}$ & $\begin{array}{c}3 \text { months } \\
\text { Follow up } \\
X \text { (SD) }\end{array}$ & F-Value & P-Value \\
\hline \multicolumn{6}{|l|}{ ACUTENS } \\
\hline Pain intensity & $8.10(1.85)$ & $5.40(2.10)$ & $\begin{array}{c}3.40 \\
(1.26)\end{array}$ & 17.53 & $0.000^{*}$ \\
\hline $\begin{array}{l}\text { Functional } \\
\text { mobility }\end{array}$ & $29.50(7.87)$ & $25.70(6.80)$ & $\begin{array}{l}27.50 \\
(5.95)\end{array}$ & 0.77 & 0.473 \\
\hline Knee AROM & $\begin{array}{l}112.50 \\
(14.90)\end{array}$ & $\begin{array}{l}121.00 \\
(12.43)\end{array}$ & $\begin{array}{c}125.90 \\
(9.00)\end{array}$ & 3.15 & 0.059 \\
\hline \multicolumn{6}{|l|}{ Acupuncture } \\
\hline Pain intensity & $7.10(1.66)$ & $2.40(1.42)$ & $\begin{array}{c}1.33 \\
(1.25)\end{array}$ & 44.64 & $0.00^{*}$ \\
\hline $\begin{array}{c}\text { Functional } \\
\text { mobility }\end{array}$ & $28.70(6.90)$ & $\begin{array}{l}12.91 \\
(5.10)\end{array}$ & $\begin{array}{c}7.20 \\
(2.25)\end{array}$ & 7.29 & $0.03^{*}$ \\
\hline Knee AROM & $\begin{array}{l}112.00 \\
(16.50)\end{array}$ & $\begin{array}{l}131.50 \\
(6.25)\end{array}$ & $\begin{array}{l}134.00 \\
(2.10)\end{array}$ & 13.73 & $0.00^{*}$ \\
\hline \multicolumn{6}{|c|}{ Control } \\
\hline Pain intensity & $8.10(1.10)$ & $7.20(1.39)$ & $\begin{array}{c}8.10 \\
(1.13)\end{array}$ & 0.22 & 0.978 \\
\hline $\begin{array}{c}\text { Functional } \\
\text { mobility }\end{array}$ & $\begin{array}{c}29.00 \\
(15.05)\end{array}$ & $\begin{array}{c}26.20 \\
(16.04)\end{array}$ & $\begin{array}{c}28.80 \\
(13.98)\end{array}$ & 1.25 & 0.301 \\
\hline Knee AROM & $\begin{array}{l}115.00 \\
(10.54)\end{array}$ & $\begin{array}{l}120.50 \\
(7.97)\end{array}$ & $\begin{array}{l}124.00 \\
(5.16)\end{array}$ & 3.06 & 0.060 \\
\hline
\end{tabular}

Table 2: Pain intensity, functional mobility and AROM of patients in all the groups at pre intervention, post intervention and at follow up assessments. 


\begin{tabular}{|c|c|c|c|c|}
\hline Variables & Groups & $\begin{array}{c}\text { Mean Difference } \\
(\mathbf{X} \boldsymbol{\Delta})\end{array}$ & SEM & p-value \\
\hline \multirow{2}{*}{ Pain intensity } & ACUTENS & 1.3 & 0.3 & \multirow{2}{*}{0.04} \\
\cline { 2 - 4 } & Acupuncture & 4.2 & 0.01 & \\
\hline \multirow{2}{*}{ Functional mobility } & ACUTENS & 4.3 & 1.12 & \multirow{2}{*}{0.01} \\
\cline { 2 - 4 } & Acupuncture & 10.6 & 3.35 & \\
\hline \multirow{2}{*}{ Knee ROM } & ACUTENS & 5.5 & 1.38 & \multirow{2}{*}{0.02} \\
\cline { 2 - 5 } & Acupuncture & 15.5 & 5.18 & \\
\hline
\end{tabular}

Table 3: Comparison of the mean difference in the pain intensity, functional mobility and knee range of motion between patients in the acupuncture and ACUTENS groups.

\section{Discussion}

This study was designed to compare the effects of acupuncture and ACUTENS on OA of the knee joints. Compared with the control group, patients that were treated with acupuncture and those who received ACUTENS experienced significantly better improvement in the form of pain relive after 8 weeks of treatment which was also maintained till 3 months after intervention. Comparison of the effect of intervention showed that patients who received acupuncture experienced better outcome in the terms of changes in pain reduction, functional mobility and knee range of movement than patients who received ACUTENS. This results concur with the findings of three previous studies [24-26]. Another study compared acupuncture and sham acupuncture ((superficial) needling at distant non-acupuncture points) and showed no difference between acupuncture and sham acupuncture [27]. Lack of significance effect of acupuncture over sham intervention could be due to the fact that acupuncture treatment was administered over a short period (three times a week for 3 weeks) in this study. In contrast, in this present study, both acupuncture and ACUTENS were administered twice weekly for 8 weeks. This procedure could have produced more potent analgesic effects than the study involving acupuncture and sham intervention. Possible therapeutic effects of acupuncture could be linked to enhancing activation of A- $\delta$ and $\mathrm{C}$ afferent fibres in muscle during needle stimulation of acupuncture points thus; signals are transmitted to the spinal cord, and via afferent pathways to the midbrain [15]. The resulting flow and integration of this information among specific brain areas will leads to a change in the perception of pain via descending pain modulatory system. Acupuncture analgesia improved the noxious descending inhibitory controls and pain gate mechanism and therefore helped to reduce the patients' pain levels [15]. The evidence for the mediation of acupuncture analgesia by endorphin is very strong, while that of the involvement of monoamines need more work to verify the possible synergism of serotonin and nor epinephrine [15]. The physiologic effects of acupuncture and ACUTENS could be link to both pain gate theory and descending pain inhibitory control at different level of the central nervous system.

Patients who received acupuncture treatment showed better improvement in the term of pain reduction, in functional mobility and knee range of motion after 8 weeks of intervention which was also maintained till 3 months after treatment, this was not the case with patients who received acupuncture-like TENS. These results might suggest that acupuncture treatment was significantly more effective than ACUTENS in the management of knee OA. Many of the previous studies involving acupuncture and ACUTENS on knee osteoarthritis reported improvement with pain reduction but it appeared that there are not many which reported improvement in functional mobility [16]. It could that in the acupuncture treatment groups, whereby needles are located at the exact acupoints along the meridians, more afferent input to activate the pain gate and descending inhibitory control from the CNS were provided than in the ACUTENS group. This perhaps likely explains why acupuncture group had better pain relieve than the ACUTENS group. It is also possible that the analgesic effects experienced by the participants in this study were not due to acupuncture and ACUTENS alone but might also be due to other peripheral and central mechanisms leading to analgesia associated with the use of NSAID's and/or perhaps other mechanisms not yet investigated.

The treatment of knee OA with either acupuncture or ACUTENS produced benefits to patients with knee $\mathrm{OA}$ in terms of pain reduction, although greater reduction in pain intensity was experienced by the patients in the acupuncture group. However, the use of ACUTENS is not without some advantages when compared with acupuncture. Advantages include omission of certain risk of using needles such as infection. Many patients have a fear of needles and might prefer ACUTENS. Perhaps the most compelling reason for ACUTENS will be the ease of use and the fact that not as much as precision is required in placement of pads over the acupuncture points as pushing the needles through the skin and muscle fibres to produce 'de qi' sensations given the large areas covered by the pad. The ease of use would likely make ACUTENS more acceptable to patients and to therapist who are not certain about the points of needle placement, depth of penetration and angle of the needle trajectory. Thus, it might be that while acupuncture treatment is recommendable for use in the clinic due to its better pain relieving effects while ACUTENS could be recommended for home use by the patient in-between treatment appointments in the clinic. Currently, it appears that data are limited on public interest, patterns of use, effects and side effects of acupuncture treatment in Nigeria. However, available reports from western countries, to a large extent portray acupuncture intervention as a cost effective and safe procedure with no serious side and/or life-threatening effects. It could be that acupuncture treatment might serve a useful complimentary treatment to physiotherapy management of knee OA and other non-invasive treatment approach in the management of numerous neuromusculoskeletal conditions if there are training opportunities to increase the local availability of acupuncture practitioners in this environment.

The small sample size might undermine the generalizability of the findings of this study to patients with osteoarthritis of the knee. Also, due to the nature of the intervention, it was not possible to blind the acupuncturists to treatment. However, assessment and analysis of data was carried out by independent researcher who was blinded to group allocations and the type of intervention administered to the patients. Using 3 acupuncture points stimulation could be another limitation of the study. Perhaps most clinicians in acupuncture practice will use more than 3 points at local and distal points a time.

\section{Conclusion}

Soft tissue manipulation combined with acupuncture and ACUTENS treatment had significant and short-term pain relieving effects when compared to STM only in patients with osteoarthritis of the knee. Acupuncture treatment produce better outcome than ACUTENS in terms of pain relief and functional mobility. 
Citation: Ganiyu S, Bello UM (2016) Comparison of the Effects of Acupuncture and Acupuncture-Like Transcutaneous Electrical Nerve Stimulation Among Adult Nigerian Adult with Osteoarthritis of the Knee. J Altern Complement Integr Med 2: 009.

\section{References}

1. Adams JC, Hamblen, DL (1990) Outline of Orthopaedics. (11thedn), Churchill Livingstone, London, UK.

2. Pomonis JD, Boulet JM, Gottshall SL, Phillips S, Sellers R, et al. (2005) Development and pharmacological characterization of a rat model of osteoarthritis pain. Pain 114: 339-346.

3. Wen DY (2000) Intra-articular hyaluronic acid injections for knee osteoarthritis. Am Fam Physician 62: 565-570.

4. Felson DT, Naimark A, Anderson J, Kazis L, Castelli W, et al. (1987) The prevalence of knee osteoarthritis in the elderly. The Framingham Osteoarthritis Study. Arthritis Rheum 30: 914-918.

5. Lawrence RC, Helmick CG, Arnett FC, Deyo RA, Felson DT, et al. (1998) Estimates of the prevalence of arthritis and selected musculoskeletal disorders in the United States. Arthritis Rheum 41: 778-799.

6. Akinpelu AO, Maduagwu SM, Odole AC, Alonge TO (2011) Prevalence and pattern of knee osteoarthritis in a North Eastern Nigerian rural community. East African Orthopaedic Journal 5: 5-12.

7. Dieppe PA (1989) Clinical Conundrum. British Journal of Rheumatology 28 242.

8. Cheing GL, Hui-Chan CW (2001) The motor dysfunction of patients with knee osteoarthritis in a Chinese population. Arthritis Rheum 45: 62-68.

9. Guccione AA, Felson DT, Anderson JJ, Anthony JM, Zhang Y, et al. (1994) The effects of specific medical conditions on the functional limitations of elders in the Framingham Study. Am J Public Health 84: 351-358.

10. Doherty M (2002) Pain in osteoarthritis. In: Giamberardino (ed.). An updated Review: Refresher Course Syllabus. International Association for the Study of Pain. Seattle, USA. Pg no: 51-57.

11. Tramèr MR, Moore RA, Reynolds DJ, McQuay HJ (2000) Quantitative estimation of rare adverse events which follow a biological progression: a new model applied to chronic NSAID use. Pain 85: 169-182.

12. American College of Rheumatology Subcommittee on Osteoarthritis Guidelines (2000) Recommendations for the medical management of osteoarthritis of the hip and knee: 2000 update. American College of Rheumatology Subcommittee on Osteoarthritis Guidelines. Arthritis Rheum 43: 1905-1915.

13. Witt C, Brinkhaus B, Jena S, Linde K, Streng A, et al. (2005) Acupuncture in patients with osteoarthritis of the knee: a randomised trial. Lancet 366 136-143.

14. Tukmachi E, Jubb R, Dempsey E, Jones $P$ (2004) The effect of acupuncture on the symptoms of knee osteoarthritis--an open randomised controlled study. Acupunct Med 22: 14-22.
15. Stux G, Pomeranz B (1998) Basics of Acupuncture. Springer Science \& Business Media, Berlin, Germany.

16. Sangdee C, Teekachunhatean S, Sananpanich K, Sugandhavesa N, Chiewchantanakit S (2002) Electroacupuncture versus diclofenac in symptomatic treatment of osteoarthritis of the knee: a randomized controlled trial. BMC Complement Altern Med 2: 3.

17. Osiri M, Welch V, Brosseau L, Shea B, McGowan J, et al. (2000) Transcutaneous electrical nerve stimulation for knee osteoarthritis. Cochrane Database Syst Rev.

18. Sokunbi OG, Usman MB (2014) Effects of Conventional and Acupuncture like Transcutaneous Electrical Nerve Stimulation on Osteoarthritis of the Knee. Nigerian Journal of Experimental and Clinical Biosciences 2: 69-74.

19. Kessler S, Guenther KP, Puhl W (1998) Scoring prevalence and severity in gonarthritis: the suitability of the Kellgren \& Lawrence scale. Clin Rheumatol 17: 205-209

20. Itoh K, Hirota S, Katsumi Y, Ochi H, Kitakoji H (2008) A pilot study on using acupuncture and Transcutaneous Electrical Nerve Stimulation (TENS) to treat knee Osteoarthritis (OA). Chin Med 3: 2

21. Von Baeyer CL, Spagrud LJ, McCormick JC, Choo E, Neville K, et al. (2009) Three new datasets supporting use of the Numerical Rating Scale (NRS-11) for children's self-reports of pain intensity. Pain 143: 223-227.

22. Watkins MA, Riddle DL, Lamb RL, Personius WJ (1991) Reliability of goniometric measurements and visual estimates of knee range of motion obtained in a clinical setting. Phys Ther 71: 90-96.

23. Nordin E, Rosendahl E, Lundin-Olsson L (2006) Timed "Up \& Go" test: reliability in older people dependent in activities of daily living--focus on cognitive state. Phys Ther 86: 646-655

24. Christensen BV, luhl IU, Vilbek H, Bülow HH, Dreijer NC, et al. (1992) Acu puncture treatment of severe knee osteoarthrosis. A long-term study. Acta Anaesthesiol Scand 36: 519-525.

25. Berman BM, Singh BB, Lao L, Langenberg P, Li H, et al. (1999) A randomized trial of acupuncture as an adjunctive therapy in osteoarthritis of the knee. Rheumatology (Oxford) 38: 346-354.

26. Tillu A, Tillu S, Vowler S (2002) Effect of acupuncture on knee function in advanced osteoarthritis of the knee: a prospective, non-randomised controlled study. Acupunct Med 20: 19-21.

27. Takeda W, Wessel J (1994) Acupuncture for the treatment of pain of osteoarthritic knees. Arthritis Care Res 7: 118-122. 